International Journal of Pure and Applied Mathematics

Volume 107 No. 4 2016, 875-887

ISSN: 1311-8080 (printed version); ISSN: 1314-3395 (on-line version)

url: http://www.ijpam.eu

doi: 10.12732/ijpam.v107i4.6

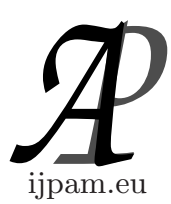

\title{
REFLECTIVE WELL-FOUNDED RELATIONS
}

\author{
Martin Dowd \\ 1613 Wintergreen Pl. \\ Costa Mesa, CA 92626, USA
}

\begin{abstract}
New axioms for set theory have already been given by the author making use of $\Sigma_{1}^{1}$ well-founded relations, whose definition "reflects" in a club (i.e., defines a well-founded relation on a club of cardinals). The method is generalized by requiring only that the definition reflect in a stationary set, for some specific stationary sets.
\end{abstract}

AMS Subject Classification: $03 e 55$

Key Words: set chains, new axioms, function chains

\section{Introduction}

In [9] a new axiom for set theory is given, stating that there are cardinals where there are set chains mod the club filter, of length $\alpha$ for any $\alpha$ which is the maximum chain length of a well-founded relation defined by a $\Sigma_{1}^{1}$ formula, which defines a well-founded relation in a club of cardinals. This relaxes the restriction on the "reflection points" to all inaccessible cardinals, of the definition of [6]. As will be seen here, it does so in a way so that longer set chains can be specified, using larger filters than the club filter.

Obviously a question of fundamental importance is whether enlarging the filter of reflection sets produces larger chain lengths. It is shown that larger heights are produced, and so if $V=L$ larger chain lengths.

The definition of $\mathrm{H}^{A}(X)$ given in [9] is not correct; a correct definition is given in section 7 .

Let Card denote the cardinals and Inac the (strongly) inaccessible cardinals. For $\kappa \in$ Card let $\operatorname{Card}_{\kappa}$ denote $\operatorname{Card} \cap \kappa$ and $\operatorname{Inac}_{\kappa} \operatorname{Inac} \cap \kappa$. Let $\operatorname{Pow}(S)$ denote

Received: March 31, 2016

Published: May 5, 2016 (c) 2016 Academic Publications, Ltd.

url: www.acadpubl.eu 
the power set of the set $S$.

\section{Universal WF's}

Recalling some standard definitions as in [9], for a well-founded relation $R$ and $A \in \operatorname{Fld}(R) \Omega(A)$ will be used to denote the rank of $A$, which is defined by a standard recursion; and $\Omega(R)$ the rank of $R$. Let $R_{A}$ be the relation $R(X, A) \wedge R(Y, A) \wedge R(X, Y)$; then $R_{A}$ is well-founded and $\Omega\left(R_{A}\right)=\Omega(A)$. If $R(A, B)$ then $\Omega(A)<\Omega(B)$ and so $\Omega\left(R_{A}\right)<\Omega\left(R_{B}\right)$.

Let $L_{\in}^{s}$ be the language of set theory with second order set variables. Suppose $\kappa \in$ Card. $V_{\kappa}$ (indeed any set) is a structure for $L_{\in}^{s}$ in a well-known manner: first order variables are interpreted as elements, second order variables as unary predicates (classes, subsets of the domain), and $\in$ as membership between elements.

A formula $\phi$ with second order free variables and parameters may be denoted $\phi(\vec{X} ; \vec{P})$ to indicate the variables and parameters. If $\phi$ is a formula let $R_{\phi}$ denote the relation it defines.

Let $\Sigma_{1}^{1}$-WF denote the set of well-founded binary relations on $\operatorname{Pow}\left(V_{\kappa}\right)$ which are definable by a $\Sigma_{1}^{1}$ formula with second order free variables and parameters.

As noted in [6], if $\phi$ is a $\Sigma_{1}^{1}$ formula in two free variables, the statement $\mathrm{WF}_{\phi}$ stating that $R_{\phi}$ is well-founded is $\Pi_{1}^{1}$. The details are standard and were omitted; a sketch will be given here. A sequence $A_{i}: i<\omega$ of classes can be coded as the single class $\left\{\langle i, x\rangle: x \in A_{i}\right\}$. The statement $\mathrm{WF}_{\phi}$ is as follows: "For all $A$ if $A$ is a sequence $A_{i}: i<\omega$ of classes then there is an $i$ such that $\neg \phi\left(A_{i+1}, A_{i}\right)$ ". The substitution $\phi\left(A_{i+1}, A_{i}\right)$ is defined by straightforward recursion on $\phi$.

Formulas of $L_{\in}^{s}$ with second-order parameters may be readily coded as classes. For a formula $\phi$ without parameters let $\ulcorner\phi\urcorner$ denote an integer coding it, in a manner so that syntactic functions are, say, primitive recursive. $\phi\left(A_{1}, \ldots, A_{k}\right)$ may be coded as $\{\langle 0, \phi\rangle\} \cup\left\{\langle i, x\rangle: x \in A_{i}\right\}$; let $\left\ulcorner\phi\left(A_{1}, \ldots, A_{k}\right)\right\urcorner$ denote this class, or simple $\ulcorner\phi\urcorner$ if it is understood that $\phi$ may have second order parameters.

Adapting results of [10] (see also [8]) there is a $\Sigma_{1}^{1}$ formula $\operatorname{Tru}(X)$ which defines the truth value predicate for $\Sigma_{1}^{1}$ sentences with parameters coded as above, in suitable structures, in particular $V_{\kappa}$ for $\kappa \in$ Card.

Theorem 1. There is a $\Pi_{1}^{1}$ formula $\mathrm{WF}(X)$ which holds for $\ulcorner\phi\urcorner$ iff $R_{\phi} \in$ $\Sigma_{1}^{1}-W F$. 
Proof. The formula is $P_{1}(X) \wedge \forall A\left(P_{2}(A) \Rightarrow \exists i \neg \operatorname{Tru}\left(X, A_{i+1}, A_{i}\right)\right)$, where $P_{1}$ restricts $X$ to be the code of a $\Sigma_{1}^{1}$ formula in two free second order variables, $P_{2}$ restricts $A$ to be a sequence of $\omega$ classes, and Tru is a suitable adaptation of the predicate for sentences noted above.

Whether WF is $\Sigma_{1}^{1}$ is a question of considerable interest.

Let $X<_{\Omega} Y$ be the predicate which is true for $\ulcorner\phi\urcorner,\ulcorner\psi\urcorner$ iff $R_{\phi}, R_{\psi}$ are well-founded and $\Omega\left(R_{\phi}\right)<\Omega\left(R_{\psi}\right)$. Clearly $<\Omega$ is well-founded.

Theorem 2. If $R \in \Sigma_{1}^{1}$-WF the map $A \mapsto R_{A}$ from $\operatorname{Fld}(R)$ to $\operatorname{Fld}(<\Omega)$ is order preserving.

Proof. This follows immediately from the facts noted above that $\Omega\left(R_{A}\right)=$ $\Omega(A)$ and $\Omega(A)<\Omega(B)$ if $R(A, B)$.

$<_{\Omega}$ may thus be called a universal WF for $\Sigma_{1}^{1}-\mathrm{WF}$.

Theorem 3. Suppose $\kappa \in$ Card and $R \in \Sigma_{1}^{1}-W F$.

a. $\Omega(R)<\Omega(<\Omega)$.

b. $<_{\Omega}$ is not $\Sigma_{1}^{1}$.

Proof. It is readily seen that $\Omega(R) \leq \Omega(<\Omega, R)$. Part a follows since $\Omega(<\Omega, R$ )$<\Omega\left(<_{\Omega}\right)$. For part b, if $<_{\Omega}$ were $\Sigma_{1}^{1}$ then $\Omega\left(<_{\Omega}\right)<\Omega\left(<_{\Omega}\right)$ would follow.

\section{Reflective WF's}

For $\kappa \in$ Card a formula $\phi(\vec{X} ; \vec{P})$ defines a predicate not only on $\operatorname{Pow}\left(V_{\kappa}\right)$ but on $\operatorname{Pow}\left(V_{\lambda}\right)$ for $\lambda \in \operatorname{Card}_{\kappa}$ as well, by restricting $X_{i}$ to range over $\operatorname{Pow}\left(V_{\lambda}\right)$ and replacing $P_{i}$ by $P_{i} \cap V_{\lambda}$. Let $R_{\phi \lambda}$ denote the relation defined by $\phi$ in $V_{\lambda}$; when $R$ denotes $R_{\phi} R_{\phi \lambda}$ may be denoted $R_{\lambda}$.

When $R \in \Sigma_{1}^{1}$-WF say that $R$ reflects in $S \subseteq \operatorname{Card}_{\kappa}$ (or $S$ is a reflection set for $R$ ) if $R_{\lambda}$ is a WF for $\lambda \in S$. Suppose $\kappa \in$ Inac. With this assumption (indeed with the weaker assumption that $\kappa$ is regular uncountable) the club filter $\mathcal{C}$ is a $\kappa$-complete normal filter on $\mathcal{C}$. Also $\operatorname{Card}_{\kappa}$ is club if $\kappa \in \operatorname{Inac}_{\text {. Say }}$ that $R$ is reflective if $R$ reflects in a stationary set of cardinals.

Theorem 4. Suppose $\kappa \in$ Inac and $\phi$ is a formula defining the binary relation $R$ in $V_{\kappa}$.

a. If $R$ is ill-founded then there is a club $C \subseteq \operatorname{Card}_{\kappa}$ such that for $\lambda \in C$, $R_{\lambda}$ is ill-founded. 
b. If there is a stationary $S \subseteq \operatorname{Card}_{\kappa}$ such that for $\lambda \in S R_{\lambda}$ is well-founded, then $R$ is well-founded.

Proof. Part a follows by theorem 11 of [6] and the $\omega$-completeness of the club filter. Part b follows immediately.

Suppose $\kappa \in$ Inac and $\mathcal{F}$ is a proper $\kappa$-complete normal filter containing $\mathcal{C}$. Let $\mathcal{F} \Sigma_{1}^{1}$-WF denote the elements $R \in \Sigma_{1}^{1}$-WF such that $R$ reflects in $S$ for some $S \in \mathcal{F}$.

Suppose $\kappa \in \operatorname{Inac}$ and $S \subseteq \operatorname{Card}_{\kappa}$ is stationary. The set $\{S \cap C: C \in \mathcal{C}\}$ generates a proper $\kappa$-complete normal filter containing $\mathcal{C} \cup\{S\}$, which will be denoted $\mathcal{C}_{S}$. $\mathcal{C}$ may be considered the special case $S=\operatorname{Card}_{\kappa} . \mathcal{C}_{I}$ will be used to denote $\mathcal{C}_{S}$ when $S=\operatorname{Inac}_{\kappa}$; this is defined only if $\operatorname{Inac}_{\kappa}$ is stationary, i.e., $\kappa$ is Mahlo.

For $X \subseteq \operatorname{Inac}_{\kappa}$ let $\mathrm{H}(X)=\{\lambda \in X: X \cap \lambda$ is a stationary subset of $\lambda\}$.

Let $\mathrm{WF}^{\mathcal{C}_{\mathcal{S}}}(X)$ be the predicate stating that $X=\ulcorner\phi\urcorner$ where $\phi$ is a $\Sigma_{1}^{1}$ formula in two free variables, such that $R_{\phi} \in \mathcal{C}_{\mathcal{S}} \Sigma_{1}^{1}$-WF.

Theorem 5. There is a $\Sigma_{1}^{1}$ formula $\theta(X ; S)$ with the following properties, where $\kappa \in \operatorname{Inac}$ and $S \subseteq \mathrm{Inac}_{\kappa}$ is stationary:

a. In $V_{\kappa}, \theta$ defines $\mathrm{WF}^{\mathcal{C}_{\mathcal{S}}}$.

b. If $\lambda \in \mathrm{Inac}_{\kappa}$ then in $V_{\lambda}, \theta$ defines $\mathrm{WF}^{\mathcal{C}}$.

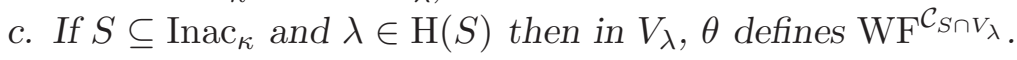

Proof. The predicate $P(X, \lambda)$ stating that $R_{\phi, \lambda}$ is well-founded is first order, uniformly in $V_{\kappa}$ for $\kappa \in$ Inac (see lemma 17 of [6] for some relevant facts). By theorem 4 , the formula $\theta$ may be stated as " $\exists C(C$ is a club of cardinals and $\forall \lambda \in C \cap S P(X, \lambda))$.

Let $<_{\Omega}^{\mathcal{C}_{S}}$ be the predicate stating that $\mathrm{WF}^{\mathcal{C}_{S}}(X)$ and $\mathrm{WF}^{\mathcal{C}_{S}}(Y)$ and $\exists C(C$ is club of cardinals and $\left.\forall \lambda \in C \cap S\left(\Omega\left(R_{\phi, \lambda}\right)<\Omega\left(R_{\psi, \lambda}\right)\right)\right)$, where $X=\ulcorner\phi\urcorner$ and $Y=\ulcorner\psi\urcorner$. Note that $\Omega\left(R_{\phi}\right)<\Omega\left(R_{\psi}\right)$ is not required; whether this follows is left as an open question. By $\omega$-completeness of $\mathcal{C}_{S},<_{\Omega}^{\mathcal{C}_{S}}$ is well-founded.

Theorem 6. $<_{\Omega}^{\mathcal{C}}$ is $\mathcal{C}_{I} \Sigma_{1}^{1}$. If $S$ is stationary then $<_{\Omega}^{\mathcal{C}_{S}}$ is $\mathcal{C}_{\mathrm{H}(S)} \Sigma_{1}^{1}$.

Proof. The predicate $Q(X, Y, \lambda)$ stating that $\Omega\left(R_{\phi, \lambda}\right)<\Omega\left(R_{\psi, \lambda}\right)$ is first order, uniformly in $V_{\kappa}$ for $\kappa \in$ Inac. The theorem now follows by the method of cases $\mathrm{b}$ and $\mathrm{c}$ of theorem 5 . 


\section{Ordinals Defined from $\mathcal{C}_{S}$}

As in [9], for a well-founded relation $R$ let $\Upsilon(R)$ denote the supremum of the lengths of the ascending chains in $R$. Unlike $\Omega, \Upsilon\left(R_{A}\right)<\Upsilon(R)$ does not follow, but only $\Upsilon(A) \leq \Upsilon(R)$; and $R(A, B)$ does not imply $\Upsilon\left(R_{A}\right)<\Upsilon\left(R_{B}\right)$, but only $\Upsilon\left(R_{A}\right) \leq \Upsilon\left(R_{B}\right)$.

Given $R \in \Sigma_{1}^{1}$-WF Let $R_{\infty}$ be the WF which adjoins an element $\infty$ to $\operatorname{Fld}(R)$, where $R_{\infty}(A, \infty)$ for all $A \in \operatorname{Fld}(R)$. This is $\Sigma_{1}^{1}$, and if $R$ is nonempty $\Omega(R)<\Omega\left(R_{\infty}\right)$. The definition can be given, so that $\left(R_{\infty}\right)_{\lambda}=\left(R_{\lambda}\right)_{\infty}$.

For $\kappa \in$ Inac and $S \subseteq$ Inac $_{\kappa}$ stationary, define the following ordinals:

$$
\begin{aligned}
& \alpha_{s \Omega}=\sup \left\{\Omega(R): R \in \Sigma_{1}^{1}-\mathrm{WF}\right\} \\
& \alpha_{s}=\sup \left\{\Upsilon(R): R \in \Sigma_{1}^{1}-\mathrm{WF}\right\} \\
& \alpha_{s \Omega \mathcal{C}_{S}}=\sup \left\{\Omega(R): R \in \mathcal{C}_{S} \Sigma_{1}^{1}-\mathrm{WF}\right\} \\
& \alpha_{s} \Upsilon \mathcal{C}_{S}=\sup \left\{\Upsilon(R): R \in \mathcal{C}_{S} \Sigma_{1}^{1}-\mathrm{WF}\right\} \\
& \alpha_{\Omega<\mathcal{C}_{S}}=\Omega\left(<_{\Omega}\right) \\
& \alpha_{\Upsilon<\mathcal{C}_{S}}=\Upsilon\left(<_{\Omega}\right)
\end{aligned}
$$

If $T \subseteq S$ then $\mathcal{C}_{S} \subseteq \mathcal{C}_{T}$, whence $\alpha_{s \Omega \mathcal{C}_{S}} \leq \alpha_{s \Omega \mathcal{C}_{T}}$ and $\alpha_{s} \Upsilon \mathcal{C}_{S} \leq \alpha_{s} \Upsilon \mathcal{C}_{T}$. Also $\alpha_{s \Omega \mathcal{C}_{S}} \leq \alpha_{s \Omega}$ and $\alpha_{s} \Upsilon \mathcal{C}_{S} \leq \alpha_{s} \Upsilon$.

Theorem 7. The ordinals just defined are limit ordinals.

Proof. It has already been observed that $\Omega(R)<\Omega\left(R_{\infty}\right)$. If $\Upsilon(R)$ is a successor ordinal then $\Upsilon(R)<\Upsilon\left(R_{\infty}\right)$. The theorem follows from these facts.

Theorem 8. Suppose $\kappa \in \operatorname{Inac}$ and $S \subseteq \operatorname{Inac}_{\kappa}$ is stationary,

$$
\begin{array}{cc}
\alpha_{s \Omega \mathcal{C}_{S}} & \leq \alpha_{\Omega<\mathcal{C}_{S}}<\alpha_{s \Omega \mathcal{C}_{\mathrm{H}(S)}} \\
\mathrm{IV}_{\mathrm{V}} & \mathrm{IV} \\
\alpha_{s \Upsilon \mathcal{C}_{S}} & \leq \alpha_{\Upsilon<\mathcal{C}_{S}} \leq \alpha_{s \Upsilon \mathcal{C}_{\mathrm{H}(S)}}
\end{array}
$$

This holds for $\mathcal{C}$, replacing $\mathcal{C}_{\mathrm{H}(S)}$ by $\mathcal{C}_{I}$.

Proof. The vertical inequalities follow because $\Omega(R) \geq \Upsilon(R)$ for any WF $R$. If $\alpha<\alpha_{s} \Upsilon \mathcal{C}_{S}$ then $\alpha+1<\alpha_{s} \Upsilon \mathcal{C}_{S}$ by theorem 7, whence there is an $R \in \mathcal{C}_{S} \Sigma_{1}^{1}$-WF with $\Omega(R) \geq \alpha+1$; there is a chain $A_{\xi}: \xi<\alpha$ in $R$, and $R_{A_{\xi}}$ is a chain in $<_{\Omega}^{\mathcal{C}_{S}}$, so $\alpha \leq \alpha \Upsilon<\mathcal{C}_{S}$. Thus, $\alpha_{s \Omega \mathcal{C}_{S}} \leq \alpha_{\Omega<\mathcal{C}_{S}}$. The proof that $\alpha_{s} \Upsilon \mathcal{C}_{S} \leq \alpha \Upsilon<\mathcal{C}_{S}$ is similar. $\alpha_{\Omega<\mathcal{C}_{S}}<\alpha_{s \Omega \mathcal{C}_{\mathrm{H}(S)}}$ follows because $\left(<_{\Omega}^{\mathcal{C}_{S}}\right)_{\infty} \in \mathcal{C}_{\mathrm{H}(S)} \Sigma_{1}^{1}$-WF. Immediately from theorem $6, \alpha \Upsilon<\mathcal{C}_{S} \leq \alpha_{s} \Upsilon \mathcal{C}_{\mathrm{H}(S)}$.

If $V=L$ more can be said, as will be shown in the next 2 sections. 


\section{Remarks on Uniform Transformations}

In [9] it is shown that if $V=L$ then a formula $\phi \in \Sigma_{1}^{1}$-WF can be transformed to a formula $\psi \in \Sigma_{1}^{1}$-WPS such that $\Omega\left(R_{\phi}\right) \leq \Omega\left(R_{\psi}\right)$. In the following section a simpler such transformation will be given. Various improvements can be made to the treatment in [9]. These are of independent interest, and introduce methods used in the following section.

Theorem 4 of [7] states that for any (parameter free) $\Delta_{0}^{1 f}$ formula $\phi$ there is a $\Delta_{0}^{0 f}$ formula $\psi$ such that $\phi \Leftrightarrow \exists \vec{F} \forall \vec{x} \psi$. The transformation is a simple (e.g. primitive recursive) function from the integer $\ulcorner\phi\urcorner$ to the integer $\ulcorner\psi\urcorner$. The free variables of $\exists \vec{F} \forall \vec{x} \psi$ are the same as those of $\phi$. The equivalence holds universally in the free variables, and uniformly in any structure for $L_{\epsilon}$.

As a result of the foregoing, a WF $R \in \mathcal{C}_{S} \Sigma_{1}^{1}$-WF may be assumed to be defined by a formula $\exists F \forall \vec{x}(\phi(\vec{F}, \vec{x}, \vec{P}, X, Y))$ where $\vec{F}$ are second order function variables, $\vec{x}$ are first order variables, $\vec{P}$ are second order set parameters, $X, Y$ are second order set variables; and $\psi$ is $\Delta_{0}^{0}$.

The recursion theorem for $L_{\kappa}$ given in [9] holds with minor adjustments in an admissible set. The reader is assumed to be familiar with the formal system KP, and the notion of a $\Delta_{1}^{\mathrm{KP}}$ function (see [2]). Formulas may have integer arguments. Theorems of PA (Peano arithmetic) regarding formulas with integer arguments may be carried out in KP.

A parameter free formula $\phi$ of $L_{\in}$ may be coded as an integer $\ulcorner\phi\urcorner$ in such a way that syntactic functions are $\Delta_{1}^{\mathrm{PA}}$; and also the free and parameter variables may be determined and linearly ordered.

For an integer $e$ let $N_{e}$ denote its numeral. For each $\vec{x}$ there is a $\Sigma_{1}$ formula $\operatorname{Tru}(n, \vec{x})$ such that for each $\Sigma_{1}$ formula $\phi(\vec{x}), \vdash_{\mathrm{KP}} \phi(\vec{x}) \Leftrightarrow \operatorname{Tru}\left(N_{\ulcorner\phi\urcorner}, \vec{x}\right)$. See proposition V.1.6 of [1].

Theorem 9. For any $\Sigma_{1}$ formula $\phi(n, \vec{x})$ with an integer argument and $k$ unrestricted arguments there is a $\Sigma_{1}$ formula $\psi(\vec{x})$ such that $\vdash_{\mathrm{KP}} \psi(\vec{x}) \Leftrightarrow$ $\phi\left(N_{\ulcorner\psi\urcorner}, \vec{x}\right)$.

Proof. let $f$ be the $\Delta_{1}^{\mathrm{PA}}$ function which maps $e=\ulcorner\phi(n, \vec{x})\urcorner$ to $\left\ulcorner\operatorname{Tru}\left(N_{e}, N_{e}, \vec{x}\right)\right\urcorner$. Let $e_{1}=\ulcorner\phi(f(n), \vec{x})\urcorner$. Let $e_{2}=f\left(e_{1}\right)$, and let $\psi(\vec{x})$ be the formula with $e_{2}=$ $\ulcorner\psi\urcorner$. Then $\psi=\operatorname{Tru}\left(N_{e_{1}}, N_{e_{1}}, \vec{x}\right) \Leftrightarrow \phi\left(f\left(N_{e_{1}}\right), \vec{x}\right) \Leftrightarrow \phi\left(N_{e_{2}}, \vec{x}\right) \Leftrightarrow \phi\left(N_{\ulcorner\psi\urcorner}, \vec{x}\right)$.

Theorem 10. Say that $\phi_{e}=\phi$ iff $\ulcorner\phi\urcorner=e$. Given $\vec{x}$, suppose $f$ is a $\Delta_{1}^{\mathrm{PA}}$ function which maps each $\ulcorner\phi(\vec{x})\urcorner$ to some $\ulcorner\psi(\vec{x})\urcorner$. Then there is an $e=\ulcorner\phi(\vec{x})\urcorner$ such that $\vdash_{\mathrm{KP}} \phi_{f}(e) \Leftrightarrow \phi_{e}$. 
Proof. Let $\phi$ be $\operatorname{Tru}(f(n), \vec{x})$, let $\psi$ be as in theorem 9, and let $e=\ulcorner\psi\urcorner$. Then $\phi_{e}(\vec{x})=\psi(\vec{x}) \Leftrightarrow \phi(\ulcorner\psi\urcorner, \vec{x}) \Leftrightarrow \operatorname{Tru}(f(\ulcorner\psi\urcorner), \vec{x})=\operatorname{Tru}(f(e), \vec{x}) \Leftrightarrow \phi_{f(e)}(\vec{x})$.

In an admissible set $A$ a formula $\phi(\vec{x} ; \vec{p})$ (or $\phi\left(p_{1}, \ldots, p_{k}\right)$ or $\phi$ ) with parameters $p_{1}, \ldots, p_{k}$ from $A$ defines a relation on $A$, which will be denoted $R_{\phi}$. Such a formula may be coded as the sequence $\left\langle\ulcorner\phi\urcorner, p_{1}, \ldots, p_{k}\right\rangle$; this may be denoted $\left\ulcorner\phi\left(p_{1}, \ldots, p_{k}\right)\right\urcorner$, or simply $\ulcorner\phi\urcorner$.

In [9] a transformation is given in $L_{\kappa}$ for $\kappa \in$ Card, from a WF to a WOS. A simpler transformation can be given. It can be given for recursively listed admissible sets, but for brevity will be stated only for $L_{\kappa}$.

Suppose $\kappa \in$ Card. Let $J_{0}$ denote the Godel pairing function. Given a formula with parameters $\phi(\gamma, \alpha, \beta ; \vec{\pi})$ let $\phi^{\#}(\alpha, \beta ; \vec{\pi})$ be defined by the following clauses:

$$
\begin{aligned}
& \phi^{\#}\left(J_{0}(\xi \cdot 2, \alpha), J_{0}(\eta \cdot 2, \beta)\right) \text { iff } \xi<\eta \vee \xi=\eta \wedge \phi(\xi, \alpha, \beta) \\
& \phi^{\#}\left(J_{0}(\xi \cdot 2, \alpha), J_{0}(\eta \cdot 2+1, \beta)\right) \text { iff } \xi \leq \eta \\
& \phi^{\#}\left(J_{0}(\xi \cdot 2+1, \alpha), J_{0}(\eta \cdot 2, \beta)\right) \text { iff } \xi<\eta \\
& \phi^{\#}\left(J_{0}(\xi \cdot 2+1, \alpha), J_{0}(\eta \cdot 2+1, \beta)\right) \text { iff } \xi<\eta
\end{aligned}
$$

It is readily seen that if $\phi_{\xi}$ is a WOS for each $\xi$, where $\phi_{\xi}(\alpha, \beta)=\phi(\xi, \alpha, \beta)$, then $\phi^{\#}$ is a WOS. If $\phi$ is $\Sigma_{1}$ then $\phi^{\#}$ is $\Sigma_{1}$.

There is an effective (e.g. $\Delta_{1}^{\mathrm{KP}}$ ) transformation $\ulcorner\phi \#=g(\ulcorner\psi\urcorner)$, which is uniform in the parameters. That is, there is a function $g_{0}$ from integers to integers such that $g(\langle\ulcorner\phi\urcorner, \vec{\pi}\rangle)=\left\langle g_{0}(\ulcorner\phi\urcorner), \vec{\pi}\right\rangle$.

Given a formula $\phi(\alpha, \beta)$ with parameters and Godel number $\eta$ and a recursive function $\theta_{e}$ let $t(e, \eta)$ be the Godel number of the formula $\exists \alpha \exists \beta \phi(\alpha, \beta) \wedge$ $\operatorname{Tru}\left(g\left(\theta_{e}\left(\left\ulcorner\phi_{\gamma}\right\urcorner\right)\right), \alpha, \beta\right)$ in the free variables $\alpha, \beta, \gamma$, where $\phi_{\gamma}$ denotes $\alpha<\gamma \wedge \beta<$ $\gamma \wedge \phi(\alpha, \beta)$.

Let $f$ be a recursive function such that $R_{f(e)}=\{\langle\eta, g(t(e, \eta))\rangle\}$. Let $e_{0}$ be such that $R_{f\left(e_{0}\right)}=R_{e_{0}}$. Let $h$ be the function where $h(\eta)=g\left(t\left(e_{0}, \eta\right)\right)$. Then $R_{e_{0}}$ is the graph of $h$. Note that $\theta_{e}$ may be given by its graph, and need not be single-valued.

Let $\psi$ be the formula with $\ulcorner\psi\urcorner=h(\ulcorner\phi\urcorner)$. If $R_{\phi}$ is empty then $\Omega\left(R_{\phi}\right) \leq$ $\Omega\left(R_{\psi}\right)$ trivially. Otherwise, this follows by induction on $\Omega\left(R_{\phi}\right)$, where the induction hypothesis is $\Omega\left(R_{\phi_{\gamma}}\right) \leq \Omega\left(R_{\psi_{\gamma}}\right)$.

Assuming $V=L$, if $R \in \mathcal{C}_{S} \Sigma_{1}^{1}$-WF has a $\Sigma_{1}^{I}$ definition derived from $\phi$ then the WPS obtained from $R_{\psi}$ is a WPS at every $\lambda$ where $R_{\lambda}$ is a WF. 


\section{Constructible Classes}

By the last remark of the previous section, it is a question of interest whether the transformations of [9] can be made uniform. A different approach will be used here. Before proceeding an oversight of [6] will be corrected. For the interpretation $I_{\epsilon}$ defined preceding theorem 7 to be applicable in $V_{\kappa}, \kappa$ must be regular; this should be added as a hypothesis to theorem 7 .

Constructible classes were considered in [12]. Some facts about them are noted in [3], which can be reformulated for the use to be made of them here.

Suppose $\kappa \in$ Inac (although as usual many facts hold if $\kappa$ is a regular uncountable cardinal). For a class $E \subseteq V_{\kappa}$ of ordered pairs write $x \tilde{\epsilon} y$ for $E(\langle x, y\rangle)$.

Lemma 11. Suppose $\kappa \in$ Inac. The following predicates on $E \subseteq V_{\kappa}$ are $\Delta_{0}^{1}$.

a. $E$ is a binary relation (a class of ordered pairs).

b. For a binary relation $E, x \in \operatorname{Fld}(E)$ and $S=\operatorname{Fld}(E)$.

c. The binary relation $E$ is well-founded.

d. The binary relation $E$ is extensional.

Proof. Straightforward. Since $\kappa$ is regular, that $E$ is well-founded may be stated as $\forall f: \omega \mapsto \operatorname{Fld}(E) \exists n(f(n+1) \tilde{\notin} f(n))$. That $E$ is extensional may be stated as $x \in \operatorname{Fld}(E) \wedge y \in \operatorname{Fld}(E) \wedge \forall w(w \tilde{\in} x \Leftrightarrow w \tilde{\epsilon} y) \Rightarrow x=y$.

Call $E$ an E-structure if it is an extensional well-founded binary relation. A formula $\phi\left(v_{1}, \ldots, v_{k}\right)$ with parameters in $\operatorname{Fld}(E)$ may be coded $\left\langle\ulcorner\phi\urcorner, v_{1}, \ldots, v_{k}\right\rangle$ where $\ulcorner\phi\urcorner$ is an integer coding the parameter free formula $\phi$.

Lemma 12. The predicate $\operatorname{Sat}(E, c)$ stating that $\phi$ is true in $E$, where $E$ is an $E$-structure and $\phi$ is a sentence with parameters in $\operatorname{Fld}(E)$, is $\Delta_{1}^{1}$.

Proof. The definition is a variation of that of $P_{2}$ given in the proof of theorem 10 of [9]. Let $P(A, E)$ be a $\Delta_{0}^{1}$ formula stating that $A$ is the class of true sentences with parameters in $\operatorname{Fld}(E)$, such that the recursion equations are satisfied by $A$. The clause for existential quantification, for example, is (roughly) $A(\langle\ulcorner\exists x \phi\urcorner, \vec{p}\rangle) \Leftrightarrow \exists x A(\langle\ulcorner\phi\urcorner, x, \vec{p}\rangle)$. The $\Sigma_{1}^{1}$ form for Sat is $\exists A P(A, E) \wedge A(x)$. The $\Pi_{1}^{1}$ form is $\forall A P(A, E) \Rightarrow A(x)$.

Theorem 13. The statement $L(X)$ that $X$ is a constructible class is a $\Sigma_{1}^{1}$ statement of $L_{\epsilon}^{s}$. 
Proof. Let $\sigma$ be the first order sentence of definition 1.16 of [12]. Then $L(X)$ may be expressed as, " $X \subseteq L$ and there are $E, x, I$ such that $E$ is a wellfounded extensional relation satisfying $\sigma, x \in \operatorname{Fld}(E)$, and $I$ is an isomorphism between $X$ and $x$ ". By an isomorphism is meant a function with domain $X$ and range $\{w \in \operatorname{Fld}(E): w \tilde{\epsilon} x\}$, such that $x \in y$ iff $I(x) \tilde{\epsilon} I(y)$.

Theorem 14. The statement $X<_{L} Y$ on the constructible classes is a $\Sigma_{1}^{1}$ statement of $L_{\in}^{s}$.

Proof. Choose an $E$ such that there are $x, y \in \operatorname{Fld}(E)$ with $X$ isomorphic to $x$ and $Y$ isomorphic to $y$. Then $X<_{L} Y$ iff $E$ satisfies the first-order statement that $x<_{L} y$.

Suppose $\kappa \in$ Card. For a class $C$ let $Q_{i}$ for $i=0,1$ denote $(C-\omega) \cup$ $\{i\} \cup\{k+2: k \in C \cap \omega\}$. For classes $A, B$ let $P(A, B)$ denote $\{\langle 0, x\rangle: x \in$ $A\} \cup\{\langle 1, x\rangle: x \in B\}$. Given a $\Sigma_{1}^{1}$ formula with parameters $\phi(C, A, B ; \vec{P})$ let $\phi^{\#}(A, B ; \vec{P})$ be defined by the following clauses:

$$
\begin{aligned}
& \phi^{\#}\left(P\left(Q_{0}(C), A\right), P\left(Q_{0}(D), B\right)\right) \text { iff } \\
& \quad C<_{L} D \vee C=D \wedge \phi(C, A, B) \\
& \phi^{\#}\left(P\left(Q_{0}(C), A\right), P\left(Q_{1}(D), B\right)\right) \text { iff } C<_{L} D \vee C=D \\
& \phi^{\#}\left(P\left(Q_{1}(C), A\right), P\left(Q_{0}(D), B\right)\right) \text { iff } C<_{L} D \\
& \phi^{\#}\left(P\left(Q_{1}(C), A\right), P\left(Q_{1}(D), B\right)\right) \text { iff } C<_{L} D
\end{aligned}
$$

It is readily seen that if $V=L$, if $\phi_{C}$ is a WOS for each $C$, where $\phi_{C}(A, B)=$ $\phi(C, A, B)$, then $\phi^{\#}$ is a WOS. If $\phi$ is $\Sigma_{1}^{1}$ then $\phi^{\#}$ is $\Sigma_{1}^{1}$.

The truth predicate for $\Sigma_{1}^{1}$ sentences with parameters of $L_{\in}^{s}$ given in section 2 is readily modified to include free variables. As in the previous section, for each $\vec{X}$ there is a $\Sigma_{1}^{1}$ formula $\operatorname{Tru}(n, \vec{X})$ such that for each parameter free $\Sigma_{1}^{1}$ formula $\phi(\vec{X}), \phi(\vec{X}) \Leftrightarrow \operatorname{Tru}\left(N_{\ulcorner\phi\urcorner}, \vec{X}\right)$ holds in $V_{\kappa}$ for $\kappa \in$ Card.

Theorems 9 and 10 hold for $\Sigma_{1}^{1}$ with appropriate modifications, with basically the same proofs. $\vdash_{\mathrm{KP}}$ is replaced by $\models_{V_{\kappa}}$ for $\kappa \in$ Card. The functions $g$ and $h$ may be adapted to this setting.

Theorem 15. Suppose $V=L, \kappa \in$ Inac, and $S \subseteq$ Inac $_{\kappa}$ is stationary. Then $\alpha_{s} \Upsilon \mathcal{C}_{S}=\alpha_{s} \Omega \mathcal{C}_{S}$.

Proof. Suppose $R$ is a $\mathcal{C}_{S} \Sigma_{1}^{1}$-WF defined by $\phi$. Let $\psi$ be the formula where $\ulcorner\psi\urcorner=h(\ulcorner\phi\urcorner)$. If $V=L, \psi$ defines a WOS $R^{\prime}$ such that $\Omega(R) \leq \Upsilon\left(R^{\prime}\right)$, and further for any $\lambda \in S$ where $R_{\lambda}$ is a WF, $\Omega\left(R_{\lambda}\right) \leq \Upsilon\left(R_{\lambda}^{\prime}\right)$.

Whether $\alpha_{s} \Upsilon \mathcal{C}=\alpha_{s \Omega \mathcal{C}}$ if $V=L$ is left open. 


\section{7. $\mathbf{H}^{A}(X)$}

For $\kappa \in$ Inac and $X, Y \subseteq \kappa$, let $X \subseteq_{\mathrm{t}} Y$ denote that $X-Y$ is thin. This relation has various well-known properties; see [5] for a list of some of these.

Lemma 16. Suppose $\kappa \in$ Inac and $\prec$ is a $\Sigma_{1}^{1}$-WF which reflects in a set $S \subseteq \operatorname{Card}_{\kappa}$. If $A \prec B$ then there is a club subset $C \subseteq \operatorname{Card}_{\kappa}$ such that for $\lambda \in C \cap S, A \cap V_{\lambda} \prec_{\lambda} B \cap V_{\lambda}$.

Proof. The proof is as in the proof of theorem 24 of [9]. Let $\vec{P}$ be the parameters in the formula $\phi$ defining $\prec$. Let $C$ be a club as in corollary 12 of [6] for the parameters $A, B, \vec{P}$.

Suppose $\kappa \in \operatorname{Inac}, S \subseteq \operatorname{Inac}_{\kappa}, \prec \in \Sigma_{1}^{1}$-WF reflects in $S, A \in \operatorname{Fld}(\prec)$, and $X \subseteq S$. Say that $\lambda \in \mathrm{H}^{A}(X)$ iff $\lambda \in X$ and $\mathrm{H}^{p}(X \cap \lambda)$ is a stationary subset of $\lambda$ for all $p \in \operatorname{Fld}\left(\prec_{\lambda}\right)$ where $p \prec_{\lambda} A \cap V_{\lambda}$. The recursion terminates when $S=\emptyset$, and $\mathrm{H}^{A}$ is the function mapping $\emptyset$ to $\emptyset$.

Theorem 17. With $\kappa, S, \prec$ as above, suppose $A \prec B$. Let $C$ be as in lemma 16. For any $X \subseteq S \cap C, \mathrm{H}^{B}(X) \subseteq \subseteq_{\mathrm{t}} \mathrm{H}\left(\mathrm{H}^{A}(X)\right)$.

Proof. This is a generalization (with corrections) of lemma 26.c of [9]; a detailed proof will be given.

First, $\mathrm{H}^{B}(X) \subseteq_{\mathrm{t}} \mathrm{H}^{A}(X)$. The proof is by induction on $\kappa$. For the basis, if $S$ is empty the claim is trivial. For arbitrary $\kappa$, for $\lambda \in S \cap C A \cap V_{\lambda} \prec_{\lambda} B \cap V_{\lambda}$. For such $\lambda$, if $\mathrm{H}^{p}(X \cap \lambda)$ is stationary for $p \prec_{\lambda} B \cap V_{\lambda}$ then using the induction hypothesis $\mathrm{H}^{p}(X \cap \lambda)$ is stationary for $p \prec_{\lambda} A \cap V_{\lambda}$.

Second, for $\lambda \in S, \mathrm{H}^{A \cap V_{\lambda}}(X \cap \lambda)=\mathrm{H}^{A}(X) \cap \lambda$. Indeed, suppose $\mu \in X \cap \lambda$. Then $\mu \in \mathrm{H}^{A \cap V_{\lambda}}(X \cap \lambda)$ iff $\mathrm{H}^{p}(X \cap \mu)$ is stationary for $p \prec_{\mu} A \cap V_{\lambda} \cap V_{\mu}$ iff $\mathrm{H}^{p}(X \cap \mu)$ is stationary for $p \prec_{\mu} A \cap V_{\mu}$ iff $\mu \in \mathrm{H}^{A}(X)$.

Suppose $\lambda \in \mathrm{H}^{B}(X)$. By the first claim, except for a thin set of $\lambda, \lambda \in$ $\mathrm{H}^{A}(X)$. Also, except for a thin set of $\lambda, A \cap V_{\lambda} \prec B \cap V_{\lambda}$, and for such $\lambda$, $\mathrm{H}^{A \cap V_{\lambda}}(X \cap \lambda)$ is stationary. By the second claim, $\mathrm{H}^{A}(X) \cap \lambda$ is stationary.

Let $\operatorname{Stat}(X)$ denote a $\Pi_{1}^{1}$ formula stating that $X$ is stationary. Let $\operatorname{Stat}_{\lambda}(x)$ denote that $x \subseteq \lambda$ is stationary.

Lemma 18. Given a formula $\phi(A, B ; \vec{P})$ of $L_{\in}^{s}$ there is a formula $\phi_{H}(\lambda, A$, $X ; S, \vec{P})$ such that for $\kappa \in$ Inac, if $\phi$ defines a $W F \prec$ in $V_{\kappa}$ which reflects in $S \subseteq \operatorname{Inac}_{\kappa}, \lambda \in S, A \in \operatorname{Fld}(\prec)$, and $X \subseteq S$, then $\models_{V_{\kappa}} \phi_{H}$ iff $\lambda \in \mathrm{H}^{A}(X)$.

Proof. Let $\operatorname{Sat}_{s}(s, f, \vec{X})$ be the $\Delta_{0}^{1}$ formula of lemma 18.a of [6], for which for a formula $\phi(\vec{X})$ of $L_{\in}^{s}$ and $\kappa \in \operatorname{Inac}, \models_{V_{\kappa}} \phi^{(s)}(\vec{X}) \Leftrightarrow \operatorname{Sat}_{s}(s,\ulcorner\phi\urcorner, \vec{X})$. 
Along the lines of lemma 19 of $[6]$, let $\phi_{w}(w, \lambda ; S, \vec{P})$ be a $\Delta_{0}^{1}$ formula stating that $\langle\mu, b, x, \nu\rangle \in w$ iff the following hold: $\lambda \in S \wedge \mu \leq \lambda \wedge \mu \in S \wedge \operatorname{Sat}_{s}\left(V_{\mu},\ulcorner b \in\right.$ $\operatorname{Fld}(\prec)\urcorner, b ; S, \vec{P}) \wedge \forall c \forall z\left(\operatorname{Sat}_{s}\left(V_{\mu},\ulcorner\phi\urcorner, c, b ; S \cap V_{\mu}, \vec{P} \cap V_{\mu}\right) \wedge z=\{\nu:\langle c, X \cap\right.$ $\left.\left.\left.V_{\mu}, z, \nu\right\rangle\right\} \Rightarrow \operatorname{Stat}_{\mu}(z)\right)$.

$\phi_{H}$ may now be given as:

$$
\begin{array}{r}
X \subseteq S \wedge \lambda \in X \wedge \exists w\left(\phi _ { w } ( w , \lambda ; S , \vec { P } ) \wedge \forall b \forall y \left(\operatorname{Sat}_{s}\left(V_{\lambda},\ulcorner\phi\urcorner, b, A \cap V_{\lambda} ; S \cap V_{\lambda}, \vec{P} \cap V_{\lambda}\right) \wedge\right.\right. \\
\left.\left.y=\left\{\mu:\left\langle b, X \cap V_{\lambda}, y, \mu\right\rangle\right\} \Rightarrow \operatorname{Stat}_{\lambda}(y)\right)\right) .
\end{array}
$$

\section{Extensible Stationary Sets}

Let $\operatorname{Ext}(S)$ be the following $\Pi_{1}^{1}$ formula, where $X=\ulcorner\phi\urcorner: \forall X \forall A\left(\mathrm{WF}_{\mathcal{C}_{S}}(X) \wedge A \in\right.$ $\left.\operatorname{Fld}\left(R_{\phi}\right) \Rightarrow \operatorname{Stat}\left(\mathrm{H}^{A}(S)\right)\right)$. For $\kappa \in \operatorname{Inac}$ say that a stationary set $S \subseteq \operatorname{Inac}_{\kappa}$ is extensible if it satisfies $\operatorname{Ext}(S)$.

For a weakly compact cardinal $\kappa$ let $\mathcal{E}$ denote the filter of enforceable sets (see e.g. [3]).

Theorem 19. For $\kappa$ weakly compact, if $S \in \mathcal{E}$ then $S$ is extensible.

Proof. Suppose $\prec$ is a $\Sigma_{1}^{1} \mathrm{WF}$. Letting $\phi_{H}$ be as in lemma 18, let $\phi_{\prec}(A)$ be the $\Pi_{1}^{1}$ formula $\forall Y\left(Y=\left\{\lambda: \phi_{H}(\lambda, S)\right\} \Rightarrow \operatorname{Stat}(Y)\right)$. It suffices to show that for $A \in \operatorname{Fld}(A), \models V_{\kappa} \Phi_{\prec}(A)$; the proof is basically the same as that of theorem 20 of [6]. Let $\phi_{\prec}^{-}(A)$ be the $\Pi_{1}^{1}$ formula $\forall B\left(B \prec A \Rightarrow \Phi_{\prec}(B)\right)$. Inductively, $\models_{V_{\kappa}} \Phi_{\prec}^{-}(A)$, and $\models V_{\kappa} \Phi_{\prec}(A)$ follows.

For a stationary set $S$ let $\operatorname{ExtP}(S)=\left\{\lambda: \models_{V_{\lambda}} \operatorname{Ext}\left(S \cap V_{\lambda}\right)\right\}$.

Theorem 20. For $\kappa$ weakly compact, if $S \in \mathcal{E}$ then $\operatorname{ExtP}(S) \in \mathcal{E}$.

Proof. It suffices to observe that if $S \in \mathcal{E}$ then by theorem $19, \models_{V_{\kappa}} \operatorname{Ext}(S)$.

For an ordinal $\alpha$ let $S_{\alpha}$ be defined by ordinal recursion as follows: $S_{0}=$ Inac, $S_{\alpha+1}=\operatorname{ExtP}\left(S_{\alpha}\right)$, and for limit $\alpha S_{\alpha}=\cap_{\beta<\alpha} S_{\beta}$. Let AxExt be the axiom $\forall \alpha \operatorname{Ext}\left(S_{\alpha}\right)$. A justification of this axiom in terms of the extendibility of the cumulative hierarchy will be outlined; as usual a more detailed discussion would be of interest.

$\operatorname{Ext}\left(S_{0}\right)$ may be justified by "paralleling" the proof of theorem 19, in a manner similar to the justification of the axiom scheme $A \preceq$ of [6], and $\mathrm{Ax} \prec$ of [9]. The axiom that Inac is stationary is justified in [4]. Inductively, assume 
$\phi_{\prec}^{-}(A)$ holds in $V_{\lambda}$ for a stationary class of $\lambda$. Since the universe is sufficiently large, there is a $\kappa \in$ Inac such that $\phi_{\prec}^{-}(A)$ holds in $V_{\lambda}$ for a stationary set $\lambda<\kappa$. In fact, there is a stationary class of such $\kappa$. Thus, there is a stationary class of $\kappa$ such that $\phi_{\prec}(A)$ holds in $V_{\kappa}$. This argument holds for any $\prec$, so Inac is an extensible class.

Assuming $\operatorname{Ext}\left(S_{\alpha}\right)$, since the universe is sufficiently large the cardinals $\kappa \in$ Inac where $\models_{V_{\kappa}} \operatorname{Ext}\left(S_{\alpha}\right)$ form a stationary class. Repeating the argument for $S_{0}, \operatorname{Ext}\left(S_{\alpha+1}\right)$.

Assuming $\operatorname{Ext}\left(S_{\beta}\right)$ for $\beta<\alpha$, since the universe is sufficiently large the cardinals $\kappa \in$ Inac where $\models_{V_{\kappa}} \forall \beta<\alpha \operatorname{Ext}\left(S_{\beta}\right)$ form a stationary class. Repeating the argument for $S_{0}, \operatorname{Ext}\left(S_{\alpha}\right)$.

Strengthening this axiom is left to future research.

\section{WF-Reflective Cardinals}

Say that a cardinal $\kappa$ is WF-reflective if every $\Sigma_{1}^{1}$-WF is reflective. By theorem 1 , If $\kappa$ is weakly compact then $\kappa$ is WF-reflective. These cardinals are of considerable interest, in particular whether their existence can be justified by extending the cumulative hierarchy, and how this question relates to the justification of weakly compact cardinals.

\section{Function Chains Mod a Filter}

Function chains were considered by the author in previous papers in the study of new axioms which extend the cumulative hierarchy. They are not necessary to this purpose in its present form. They are of independent interest, though, and some properties will be given here.

Suppose $\kappa \in$ Inac (although many facts hold in a regular uncountable cardinal). Let $\mathcal{N}$ denote the set of functions $f: \kappa \mapsto \kappa$. For $f_{1}, f_{2} \in \mathcal{N}$ and $S \subseteq \kappa$ say that $f_{1}<_{S} f_{2}$ if $f_{1}(\alpha)<f_{2}(\alpha)$ for $\alpha \in S$. The notions $f_{1} \leq_{S} f_{2}$ and $f_{1}=S f_{2}$ are defined similarly.

Suppose $\mathcal{F}$ is a filter on $\kappa$. As in definition 24.4 of [11] say that $f_{1}<_{\mathcal{F}} f_{2}$ if $f_{1}<_{S} f_{2}$ for some $S \in \mathcal{F}$, and similarly for $f_{1} \leq_{\mathcal{F}} f_{2}$ and $f_{1}=_{\mathcal{F}} f_{2}$. These notions have the following readily verified properties:

$<_{\mathcal{F}}$ is transitive, and if $\mathcal{F}$ is $\omega$-complete it is well-founded.

$\leq_{\mathcal{F}}$ is reflexive and transitive, and $=_{\mathcal{F}}$ is its quotient equivalence relation.

$=_{\mathcal{F}}$ is a congruence relation for $<_{\mathcal{F}}$ and $\leq_{\mathcal{F}}$. 
A partial function $f$ defined on a domain $S \in \mathcal{F}$ may be extended arbitrarily to $\kappa$. The relations $<_{\mathcal{F}}, \leq_{\mathcal{F}}$, and $=_{\mathcal{F}}$ between two such extensions depend only on the partial functions.

Suppose $\mathcal{F}$ is a proper $\kappa$-complete normal filter containing $\mathcal{C}$. A chain in $<\mathcal{F}$ will be called a function chain $\bmod \mathcal{F}$. Suppose $\prec \in \mathcal{F} \Sigma_{1}^{1}$-WF reflects in a stationary set $S$. For each $A \in \operatorname{Fld}(\prec)$ let $f_{A}: S \mapsto \kappa$ be the function where $f_{A}(\lambda)=\Omega\left(\prec_{\lambda, A \cap V_{\lambda}}\right)$. By lemma 16 if $A \prec B$ then $f_{A}<_{\mathcal{F}} f_{B}$. Thus, there are function chains $\bmod \mathcal{F}$ of length $\alpha$ for any $\alpha<\Upsilon(\prec)$.

\section{References}

[1] J. Barwise, Admissible Sets and Structures, Springer-Verlag, Berlin, 1971.

[2] K. Devlin, Constructibility, Springer-Verlag (1984).

[3] M. Dowd, Iterating Mahlo's operation, Int. J. Pure Appl. Math. bf 9, no. 4 (2003), 469-512.

http://www.hyperonsoft.com/imol.pdf

[4] M. Dowd, Some New Axioms for Set Theory, Int. J. Pure Appl. Math. 66, no. 2 (2011), 121-136.

http://www.ijpam.eu/contents/2011-66-2/1/1.pdf

[5] M. Dowd, A Lower Bound on the Mahlo Rank of a Weakly Compact Cardinal, Int. J. Pure Appl. Math. 68, no. 4 (2011), 415-438.

http://www.ijpam.eu/contents/2011-68-4/4/4.pdf

[6] M. Dowd, Function Chains from Uniform Sigma-1-1 Well Orders, Int. J. Pure Appl. Math. 93 no. 5 (2014), 661-684.

http://dx.doi.org/10.12732/ijpam.v93i5.7

[7] M. Dowd, Pi-1-1 Normal Form in a Regular cardinal, Int. J. Pure Appl. Math. 96, no. 1 (2014), 135-142. http://dx.doi.org/10.12732/ijpam.v96i1.10

[8] M. Dowd, Downsets and Generalized Descriptive Set Theory, Int. J. Pure Appl. Math. 101, no. 2 (2015), 305-324. http://dx.doi.org/10.12732/ijpam.v101i2.15

[9] M. Dowd, Sigma-1-1 Well-Founded Relations and Set Chains, Int. J. Pure Appl. Math. to appear.

[10] F. Drake, Set Theory, An Introduction to Large Cardinals, North Holland (1974).

[11] T. Jech, Set Theory, Springer, 2003.

[12] L. Tharp, Constructability in impredicative set theory, Ph. D. Thesis, MIT (1965). 
